\title{
Characterization of anisotropic nanopore structure of organic-rich marine shales in China: A SANS study
}

\author{
Rui Zhanga, Yang Wang ${ }^{\mathrm{b}, \mathrm{c}}$ and Shimin Liu ${ }^{\mathrm{a}}$
}

aDepartment of Energy and Mineral Engineering, $\mathrm{G}^{3}$ Center and Energy Institute, Pennsylvania State University, University Park, Pennsylvania 16802, USA, zhangrui117@gmail.com and szl3@psu.edu

${ }^{b}$ Key Laboratory of Coalbed Methane Resources and Reservoir Formation Process, Ministry of Education, Xuzhou, Jiangsu 221008, China, wangycumt@163.com

'School of Resources and Earth Science, China University of Mining and Technology, Xuzhou, Jiangsu 221116, China

Shale nanopore structure is a critical factor in controlling methane adsorption and diffusion, and migration mechanisms of hydrocarbons. Small-angle neutron scattering (SANS) was employed to characterize the anisotropic nanopore structure of organic-rich marine shales from the Longmaxi Formation in China. Quantitatively, we used the alignment factor and Hermans' orientation function to determine the degree of anisotropy based on the azimuthal scattering profiles (Fig. 1). For the sampling location $L$, the thin sections cut parallel to the bedding have a tiny degree of anisotropy. With increasing pore size, the degree of anisotropy rapidly increases within pore size of $30 \AA$, decreases and increases within the pore range of 30-400 $\AA$, and slightly decreases at the pore size $>400 \AA$ for the thin sections cut perpendicular to the bedding. However, for the sampling location $\mathrm{W}$, both the thin sections cut perpendicular and parallel to the bedding have an apparent degree of anisotropy. Furthermore, focused on the thin sections cut perpendicular to the bedding, we found that the degree of anisotropy may positively correlate with thermal maturity of organic matter.

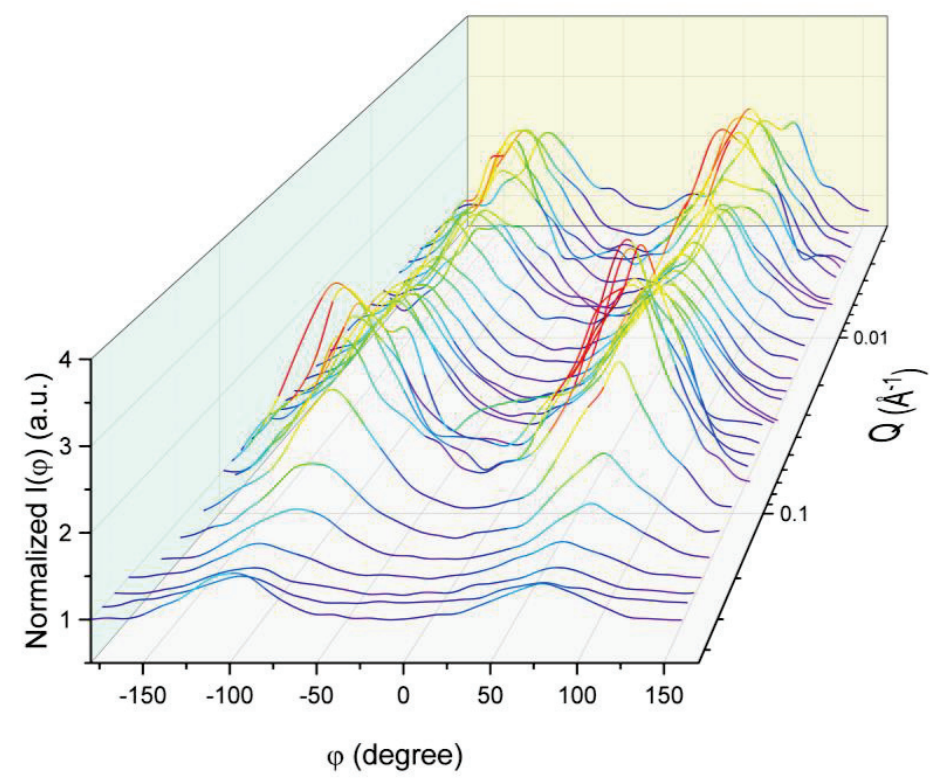

Fig. 1. Anisotropic scattering profiles.

Acknowledgments

We want to thank Lilin He for the help of SANS measurement and data reduction. This research used resources at the High Flux Isotope Reactor, a DOE Office of Science User Facility operated by the Oak Ridge National Laboratory. 